\title{
A family of two-variable derivative polynomials for tangent and secant
}

\author{
Shi-Mei Ma* \\ School of Mathematics and Statistics \\ Northeastern University at Qinhuangdao \\ Hebei 066004, P.R. China \\ shimeima@yahoo.com.cn
}

Submitted: May 10, 2012; Accepted: Jan 4, 2013; Published: Jan 21, 2013

Mathematics Subject Classifications: 05A05, 05A15

\begin{abstract}
In this paper we introduce a family of two-variable derivative polynomials for tangent and secant. Generating functions for the coefficients of this family of polynomials are studied. In particular, we establish a connection between these generating functions and Eulerian polynomials.
\end{abstract}

Keywords: Derivative polynomials; Eulerian polynomials; Tangent function; Secant function

\section{Introduction}

Throughout this paper, denote by $D$ the differential operator $\frac{d}{d x}$. Let $y=\tan (x)$, and let $z=\sec (x)$. Then $D(y)=z^{2}$ and $D(z)=y z$. An important tangent identity is given by

$$
1+y^{2}=z^{2}
$$

In 1995, Hoffman [9] considered two sequences of derivative polynomials defined respectively by

$$
D^{n}(y)=P_{n}(y) \quad \text { and } \quad D^{n}(z)=z Q_{n}(y)
$$

for $n \geqslant 0$. From the chain rule it follows that the polynomials $P_{n}(u)$ satisfy $P_{0}(u)=u$ and $P_{n+1}(u)=\left(1+u^{2}\right) P_{n}^{\prime}(u)$, and similarly $Q_{0}(u)=1$ and $Q_{n+1}(u)=\left(1+u^{2}\right) Q_{n}^{\prime}(u)+u Q_{n}(u)$. The first few of the polynomials $P_{n}(u)$ are

$$
P_{1}(u)=1+u^{2}, P_{2}(u)=2 u+2 u^{3}, P_{3}(u)=2+8 u^{2}+6 u^{4} .
$$

*Supported by the National Natural Science Foundation of China (11126217). 
There is a wealth of literature on derivative polynomials (see [3, 4, 8, 10, 11, 14, 15] for instance).

Let $[n]=\{1,2, \ldots, n\}$, and let $\mathfrak{S}_{n}$ denote the the set of permutations of $[n]$. A permutation $\pi=\pi(1) \pi(2) \cdots \pi(n) \in \mathfrak{S}_{n}$ is alternating if $\pi(1)>\pi(2)<\cdots \pi(n)$. In other words, $\pi(i)<\pi(i+1)$ if $i$ is even and $\pi(i)>\pi(i+1)$ if $i$ is odd. It is well known [1] that the Euler numbers $E_{n}$ defined by

$$
y+z=\sum_{n=0}^{\infty} E_{n} \frac{x^{n}}{n !}
$$

count alternating permutations in $\mathfrak{S}_{n}$. The study of Euler numbers is a topic in combinatorics (see [18]). Since the tangent is an odd function and the secant is an even function, we have

$$
y=\sum_{n=0}^{\infty} E_{2 n+1} \frac{x^{2 n+1}}{(2 n+1) !} \quad \text { and } \quad z=\sum_{n=0}^{\infty} E_{2 n} \frac{x^{2 n}}{(2 n) !} .
$$

For this reason $E_{2 n+1}$ is called a tangent number and $E_{2 n}$ is called a secant number.

Let $S(x)=y+z$. Clearly, $S(0)=1$. It is easy to verify that

$$
2 D(S(x))=1+S^{2}(x) .
$$

Differentiation of (1) gives

$$
2^{2} D^{2}(S(x))=2 S(x)+2 S^{3}(x) .
$$

A second differentiation gives $2^{3} D^{3}(S(x))=2+8 S^{2}(x)+6 S^{4}(x)$. Now we present a connection between $S(x)$ and $P_{n}(u)$

Proposition 1. For $n \geqslant 0$, we have $2^{n} D^{n}(S(x))=P_{n}(S(x))$.

Proof. We proceed by induction on $n$. It suffices to consider the case $n \geqslant 3$. Assume that the statement is true for $n=k$. Then

$$
\begin{aligned}
2^{k+1} D^{k+1}(S(x)) & =2 D\left(P_{k}(S(x))\right) \\
& =2 P_{k}^{\prime}(S(x)) D(S(x)) \\
& =\left(1+S^{2}(x)\right) P_{k}^{\prime}(S(x)) \\
& =P_{k+1}(S(x)) .
\end{aligned}
$$

Thus the statement is true for $k+1$, as desired.

Writing the derivative polynomials in terms of $y$ and $z$ as follows:

$$
D^{n}(y)=\sum_{k=0}^{\left\lfloor\frac{n-1}{2}\right\rfloor} W_{n, k} y^{n-2 k-1} z^{2 k+2},
$$




$$
D^{n}(z)=\sum_{k=0}^{\left\lfloor\frac{n}{2}\right\rfloor} W_{n, k}^{l} y^{n-2 k} z^{2 k+1},
$$

we observed that the coefficients $W_{n, k}$ and $W_{n, k}^{l}$ have simple combinatorial interpretations (see [14]). The coefficient $W_{n, k}$ is the number of permutations in $\mathfrak{S}_{n}$ with $k$ interior peaks, where an interior peak of $\pi$ is an index $2 \leqslant i \leqslant n-1$ such that $\pi(i-1)<\pi(i)>\pi(i+1)$. The coefficient $W_{n, k}^{l}$ is the number of permutations in $\mathfrak{S}_{n}$ with $k$ left peaks, where a left peak of $\pi$ is either an interior peak or else the index 1 in the case $\pi(1)>\pi(2)$ (see [6] for instance).

This paper is organized as follows. In Section 2, we collect some notation, definitions and results that will be needed in the rest of the paper. In Section 3, we establish a connection between the Eulerian numbers and the expansion of $(D y)^{n}(y)$. In Section 4 . we establish a connection between the Eulerian numbers of type $B$ and the expansion of $(D y)^{n}(z)$. In Section 5, some polynomials related to $(y D)^{n}(y)$ and $(y D)^{n}(z)$ are studied.

\section{Preliminaries}

A descent of a permutation $\pi \in \mathfrak{S}_{n}$ is a position $i$ such that $\pi(i)>\pi(i+1)$. Denote by $\operatorname{des}(\pi)$ the number of descents of $\pi$. Then the equations

$$
A_{n}(x)=\sum_{\pi \in \mathfrak{S}_{n}} x^{\operatorname{des}(\pi)+1}=\sum_{k=1}^{n} A(n, k) x^{k},
$$

define the Eulerian polynomials $A_{n}(x)$ and the Eulerian numbers $A(n, k)$. Set $A_{0}(x)=1$. The exponential generating function for $A_{n}(x)$ is

$$
A(x, t)=\sum_{n \geqslant 0} A_{n}(x) \frac{t^{n}}{n !}=\frac{1-x}{1-x e^{t(1-x)}} .
$$

The numbers $A(n, k)$ satisfy the recurrence relation

$$
A(n+1, k)=k A(n, k)+(n-k+2) A(n, k-1)
$$

with the initial conditions $A(0,0)=1$ and $A(0, k)=0$ for $k \geqslant 1$ (see [17, A008292]). The first few of the Eulerian polynomials $A_{n}(x)$ are

$$
A_{0}(x)=1, A_{1}(x)=x, A_{2}(x)=x+x^{2}, A_{3}(x)=x+4 x^{2}+x^{3} .
$$

An explicit formula for $A(n, k)$ is given as follows:

$$
A(n, k)=\sum_{i=0}^{k}(-1)^{i}\left(\begin{array}{c}
n+1 \\
i
\end{array}\right)(k-i)^{n} .
$$


The hyperoctahedral group $B_{n}$ is the group of signed permutations of the set $\pm[n]$ such that $\pi(-i)=-\pi(i)$ for all $i$, where $\pm[n]=\{ \pm 1, \pm 2, \ldots, \pm n\}$. Let

$$
B_{n}(x)=\sum_{k=0}^{n} B(n, k) x^{k}=\sum_{\pi \in B_{n}} x^{\operatorname{des}_{B}(\pi)},
$$

where

$$
\operatorname{des}_{B}=\#\{i \in\{0,1,2, \ldots, n-1\} \mid \pi(i)>\pi(i+1)\},
$$

with $\pi(0)=0$. The polynomial $B_{n}(x)$ is called an Eulerian polynomial of type $B$, while $B(n, k)$ is called an Eulerian number of type $B$ (see [17, A060187]). The first few of the polynomials $B_{n}(x)$ are

$$
B_{0}(x)=1, B_{1}(x)=1+x, B_{2}(x)=1+6 x+x^{2}, B_{3}(x)=1+23 x+23 x^{2}+x^{3} .
$$

The numbers $B(n, k)$ satisfy the recurrence relation

$$
B(n+1, k)=(2 k+1) B(n, k)+(2 n-2 k+3) B(n, k-1),
$$

with the initial conditions $B(0,0)=1$ and $B(0, k)=0$ for $k \geqslant 1$. An explicit formula for $B(n, k)$ is given as follows:

$$
B(n, k)=\sum_{i=0}^{k}(-1)^{i}\left(\begin{array}{c}
n+1 \\
i
\end{array}\right)(2 k-2 i+1)^{n}
$$

for $0 \leqslant k \leqslant n$ (see [7] for details).

For $n \geqslant 0$, we always assume that

$$
\begin{aligned}
& (D y)^{n+1}(y)=(D y)(D y)^{n}(y)=D\left(y(D y)^{n}(y)\right), \\
& (D y)^{n+1}(z)=(D y)(D y)^{n}(z)=D\left(y(D y)^{n}(z)\right), \\
& (y D)^{n+1}(y)=(y D)(y D)^{n}(y)=y D\left((y D)^{n}(y)\right), \\
& (y D)^{n+1}(z)=(y D)(y D)^{n}(z)=y D\left((y D)^{n}(z)\right) .
\end{aligned}
$$

Clearly, $(D y)^{n}(y+z)=(D y)^{n}(y)+(D y)^{n}(z)$. For $n \geqslant 1$, we define

$$
(D y)^{n}(y+z)=\sum_{k=0}^{2 n} J(2 n, k) y^{2 n-k} z^{k+1} .
$$

In Section 3 and Section 4 , we respectively obtain that

$$
J(2 n, 2 k-1)=2^{n} A(n, k), \quad 1 \leqslant k \leqslant n,
$$

and

$$
J(2 n, 2 k)=B(n, k), \quad 0 \leqslant k \leqslant n .
$$

Let $J_{n}(x)=\sum_{k=0}^{2 n} J(2 n, k) x^{k}$ for $n \geqslant 1$. Then $x J_{n}(x)=2^{n} A_{n}\left(x^{2}\right)+x B_{n}\left(x^{2}\right)$. Therefore, from [14, Theorem 3], we have

$$
x J_{n}(x)=(1+x)^{n+1} A_{n}(x) .
$$

Using (6), we get the following proposition. 
Proposition 2. For $n \geqslant 1$, we have

$$
(D y)^{n}(y+z)=(y+z)^{n+1} \sum_{k=1}^{n} A(n, k) y^{n-k} z^{k} .
$$

\section{On the expansion of $(D y)^{n}(y)$}

For $n \geqslant 1$, we define

$$
(D y)^{n}(y)=\sum_{k=1}^{n} E(n, k) y^{2 n-2 k+1} z^{2 k}
$$

Theorem 3. For $1 \leqslant k \leqslant n$, we have $E(n, k)=2^{n} A(n, k)$.

Proof. Note that $D\left(y^{2}\right)=2 y z^{2}$. Then $E(1,1)=2 A(1,1)$. Since

$$
D\left(y(D y)^{n}(y)\right)=2 \sum_{k=1}^{n} k E(n, k) y^{2 n-2 k+3} z^{2 k}+2 \sum_{k=1}^{n}(n-k+1) E(n, k) y^{2 n-2 k+1} z^{2 k+2},
$$

there follows

$$
E(n+1, k)=2(k E(n, k)+(n-k+2) E(n, k-1)) .
$$

By comparing (4) with (8), we obtain the desired result.

Let

$$
F_{n}(y)=(D y)^{n}(y)=\sum_{k=0}^{n} F(n, k) y^{2 k+1} .
$$

Then $F_{n+1}(y)=D\left(y F_{n}(y)\right)$. Hence

$$
F_{n+1}(y)=\left(1+y^{2}\right) F_{n}(y)+y\left(1+y^{2}\right) F_{n}^{\prime}(y)
$$

with initial value $F_{0}(y)=y$. Set $F_{n}(y)=2^{n} a_{n}(y)$ and $a_{n}(y)=\sum_{k=0}^{n} a(n, k) y^{2 k+1}$. It follows from Theorem 3 that

$$
a_{n}(y)=\sum_{k=1}^{n} A(n, k) y^{2 n-2 k+1}\left(1+y^{2}\right)^{k}
$$

Equating the coefficients of $y^{2 n-2 k+1}$ on both sides of $(10)$, we obtain

$$
a(n, n-k)=\sum_{i=k}^{n}\left(\begin{array}{l}
i \\
k
\end{array}\right) A(n, i)
$$

It follows from $(9)$ that

$$
a(n+1, k)=(k+1) a(n, k)+k a(n, k-1) .
$$


Let $W_{n}(x)=\sum_{k=0}^{n} a(n, k) x^{k+1}$. It is easy to verify that the polynomials $W_{n}(x)$ satisfy

$$
W_{n+1}(x)=\left(x+x^{2}\right) W_{n}^{\prime}(x),
$$

with initial value $W_{0}(x)=x$. The triangular array $\{a(n, k)\}_{n \geqslant 0,0 \leqslant k \leqslant n}$ is called a Worpitzky triangle (see [17, A028246]).

In view of (11), it is natural to consider the expansion of the operator $\left(\left(x+x^{2}\right) D\right)^{n}$. We define

$$
\left(\left(x+x^{2}\right) D\right)^{n}=\sum_{k=1}^{n} G_{n, k}(x)\left(x+x^{2}\right)^{k} D^{k}
$$

for $n \geqslant 1$. Applying the operator $\left(x+x^{2}\right) D$ on the left of $(12)$, we get

$$
G_{n+1, k}(x)=k(1+2 x) G_{n, k}(x)+\left(x+x^{2}\right) D\left(G_{n, k}(x)\right)+G_{n, k-1}(x) .
$$

On the other hand, since

$$
D^{k}\left(\left(x+x^{2}\right) D\right)=\left(x+x^{2}\right) D^{k+1}+k(1+2 x) D^{k}+k(k-1) D^{k-1},
$$

applying the operator $\left(x+x^{2}\right) D$ on the right of $(12)$, we get

$$
G_{n+1, k}(x)=k(1+2 x) G_{n, k}(x)+k(k+1)\left(x+x^{2}\right) G_{n, k+1}(x)+G_{n, k-1}(x) .
$$

By comparing (13) with (14), we obtain $D\left(G_{n, k}(x)\right)=k(k+1) G_{n, k+1}(x)$. Thus

$$
G_{n, k}(x)=\frac{1}{k !(k-1) !} D^{k-1}\left(G_{n, 1}(x)\right) .
$$

Thus $\operatorname{deg} G_{n, k}(x)=n-k$. Set $G_{n}(x)=G_{n, 1}(x)$. Then 13 reduces to

$$
G_{n+1}(x)=(1+2 x) G_{n}(x)+\left(x+x^{2}\right) D\left(G_{n}(x)\right)
$$

with initial value $G_{1}(x)=1$. Let $G_{n}(x)=\sum_{k=1}^{n} G(n, k) x^{k-1}$. It is easy to verify that

$$
G(n+1, k)=k G(n, k)+k G(n, k-1)
$$

with initial value $G(1,1)=1$. Recall that the Stirling numbers of the second kind $S(n, k)$ satisfy the recurrence relation

$$
S(n+1, k)=k S(n, k)+S(n, k-1)
$$

with initial conditions $S(0,0)=1$ and $S(n, 0)=0$ for $n \geqslant 1$ (see [17, A008277]). By comparing (15) with (16), we immediately get the following result.

Proposition 4. For $1 \leqslant k \leqslant n$, we have $G(n, k)=k ! S(n, k)$. 


\section{On the expansion of $(D y)^{n}(z)$}

For $n \geqslant 0$, we define

$$
(D y)^{n}(z)=\sum_{k=0}^{n} H(n, k) y^{2 n-2 k} z^{2 k+1}
$$

Theorem 5. For $0 \leqslant k \leqslant n$, we have $H(n, k)=B(n, k)$.

Proof. Clearly, $H(0,0)=1$. Note that $D(y z)=y^{2} z+z^{3}$. Then $H(1,0)=B(1,0)$ and $H(1,1)=B(1,1)$. Note that

$$
(D y)(D y)^{n}(z)=\sum_{k=0}^{n}(1+2 k) H(n, k) y^{2 n-2 k+2} z^{2 k+1}+\sum_{k=0}^{n}(2 n-2 k+1) H(n, k) y^{2 n-2 k} z^{2 k+3}
$$

Then

$$
H(n+1, k)=(1+2 k) H(n, k)+(2 n-2 k+3) H(n, k-1) .
$$

Hence the numbers $H(n, k)$ satisfy the same recurrence relation and initial conditions as $B(n, k)$, so they agree.

Let $(D y)^{n}(z)=z f_{n}(y)$. Using $(D y)^{n+1}(z)=D\left(y z f_{n}(y)\right)$, we get

$$
f_{n+1}(y)=\left(1+2 y^{2}\right) f_{n}(y)+y\left(1+y^{2}\right) f_{n}^{\prime}(y)
$$

with initial value $f_{0}(y)=1$.

Set $f_{n}(y)=\sum_{k=0}^{n} f(n, k) y^{2 k}$. By (17), we obtain

$$
f(n+1, k)=(1+2 k) f(n, k)+2 k f(n, k-1)
$$

for $0 \leqslant k \leqslant n$, with initial conditions $f(0,0)=1, f(0, k)=0$ for $k \geqslant 1$. It should be noted that

$$
(f(n, 0), f(n, 1), \ldots, f(n, n))
$$

is the $f$-vector of the simplicial complex dual to the permutohedra of type $B$ of rank $n$ (see [17, A145901]).

\section{$5 \quad$ Polynomials related to $(y D)^{n}(y)$ and $(y D)^{n}(z)$}

For $n \geqslant 1$, we define

$$
\begin{gathered}
(y D)^{n}(y)=\sum_{k=1}^{n} M(n, k) y^{2 k-1} z^{2 n-2 k+2}, \\
(y D)^{n}(z)=\sum_{k=1}^{n} N(n, k) y^{2 k} z^{2 n-2 k+1} .
\end{gathered}
$$


Theorem 6. For $1 \leqslant k \leqslant n$, we have

$$
\begin{gathered}
M(n+1, k)=(2 k-1) M(n, k)+(2 n-2 k+4) M(n, k-1), \\
N(n+1, k)=2 k N(n, k)+(2 n-2 k+3) N(n, k-1) .
\end{gathered}
$$

Proof. Note that

$$
(y D)(y D)^{n}(y)=\sum_{k=1}^{n}(2 k-1) M(n, k) y^{2 k-1} z^{2 n-2 k+4}+\sum_{k=1}^{n}(2 n-2 k+2) M(n, k) y^{2 k+1} z^{2 n-2 k+2} .
$$

Thus we obtain (18). Similarly, we get (19).

From (18) and (19), we immediately get a connection between $M(n, k)$ and $N(n, k)$.

Corollary 7. For $1 \leqslant k \leqslant n$, we have $M(n, k)=N(n, n-k+1)$.

Let $M_{n}(x)=\sum_{k=1}^{n} M(n, k) x^{k}$, and let $N_{n}(x)=\sum_{k=1}^{n} N(n, k) x^{k}$. Then we have

$$
M_{n}(x)=x^{n+1} N_{n}\left(\frac{1}{x}\right) .
$$

Set

$$
R_{n}(y)=(y D)^{n}(y)=\sum_{k=0}^{n} R(n, k) y^{2 k+1}, z T_{n}(y)=(y D)^{n}(z)=z \sum_{k=1}^{n} T(n, k) y^{2 k} .
$$

It is easy to verify that

$$
\begin{gathered}
R_{n+1}(y)=y\left(1+y^{2}\right) R_{n}^{\prime}(y), \\
T_{n+1}(y)=y^{2} T_{n}(y)+y\left(1+y^{2}\right) T_{n}^{\prime}(y) .
\end{gathered}
$$

Equating the coefficient of $y^{2 k+1}$ on both sides of $(21)$, we get

$$
R(n+1, k)=(2 k+1) R(n, k)+(2 k-1) R(n, k-1) .
$$

Equating the coefficient of $y^{2 k}$ on both sides of 22 , we get

$$
T(n+1, k)=2 k T(n, k)+(2 k-1) T(n, k-1) .
$$

Clearly, $R(n, n)=T(n, n)=(2 n-1) !$ !, where $(2 n-1) ! !$ is the double factorial number. It should be noted that the triangular arrays $\{R(n, k)\}_{n \geqslant 1,0 \leqslant k \leqslant n}$ and $\{T(n, k)\}_{n \geqslant 1,1 \leqslant k \leqslant n}$ are both Galton triangles (see [17, A187075]), which has been studied by Neuwirth [16]. Now we present the following result.

Theorem 8. For $n \geqslant 1$, we have

$$
R_{n}(y)=y^{2 n+1} N_{n}\left(\frac{1+y^{2}}{y^{2}}\right) \quad \text { and } \quad T_{n}(y)=\left(1+y^{2}\right)^{n} N_{n}\left(\frac{y^{2}}{1+y^{2}}\right) .
$$


Proof. Recall that $z^{2}=y^{2}+1$. Then

$$
R_{n}(y)=\sum_{k=1}^{n} M(n, k) y^{2 k-1}\left(y^{2}+1\right)^{n-k+1}=y^{-1}\left(1+y^{2}\right)^{n+1} M_{n}\left(\frac{y^{2}}{1+y^{2}}\right)
$$

and

$$
T_{n}(y)=\sum_{k=1}^{n} N(n, k) y^{2 k}\left(y^{2}+1\right)^{n-k}=\left(1+y^{2}\right)^{n} N_{n}\left(\frac{y^{2}}{1+y^{2}}\right) .
$$

It follows from $(20)$ that

$$
\left(1+y^{2}\right)^{n+1} M_{n}\left(\frac{y^{2}}{1+y^{2}}\right)=y^{2 n+2} N_{n}\left(\frac{1+y^{2}}{y^{2}}\right),
$$

as desired.

From Theorem 8, we get $R_{n}(1)=N_{n}(2)$ and $T_{n}(1)=2^{n} N_{n}\left(\frac{1}{2}\right)$. It follows from 19 that

$$
N_{n+1}(x)=(2 n+1) x N_{n}(x)+2 x(1-x) N_{n}^{\prime}(x)
$$

with initial value $N_{0}(x)=1$. The first few of the polynomials $N_{n}(x)$ are

$$
N_{1}(x)=x, N_{2}(x)=2 x+x^{2}, N_{3}(x)=4 x+10 x^{2}+x^{3} .
$$

In particular, $N(n, 1)=2^{n-1}, N(n, n)=1$ and $N_{n}(1)=(2 n-1)$ !! for $n \geqslant 1$. There is a nice description of the polynomials $N_{n}(x)$ (see [17, A156919]): if $\vartheta=2 x D$ and $r(x)=(1-x)^{-\frac{1}{2}}$, then

$$
\vartheta^{n}(r(x))=N_{n}(x) r(x)^{2 n+1} .
$$

In the following discussion, we consider some properties of the polynomials $N_{n}(x)$.

The numbers $N(n, k)$ arise often in combinatorics and other branches of mathematics (see [12 for instance). A perfect matching of [2n] is a partition of [2n] into $n$ blocks of size 2. Analyzing the placement of $2 n-1$ and $2 n$, it is easy to verify that the number $N(n, k)$ counts perfect matchings of $[2 n]$ with the restriction that only $k$ matching pairs have odd smaller entries (see [17, A185411]).

For $n \geqslant 1$, an explicit formula for $N_{n}(x)$ is given as follows (see [17, A156919]):

$$
N_{n}(x)=\sum_{k=1}^{n} 2^{n-2 k}\left(\begin{array}{c}
2 k \\
k
\end{array}\right) k ! S(n, k) x^{k}(1-x)^{n-k}
$$

where $S(n, k)$ is the Stirling number of the second kind. It follows from (24) that

$$
N(n, k)=\sum_{i=1}^{k}(-1)^{k-i} 2^{n-2 i}\left(\begin{array}{c}
2 i \\
i
\end{array}\right)\left(\begin{array}{c}
n-i \\
k-i
\end{array}\right) i ! S(n, i) .
$$

Let

$$
N(x, t)=\sum_{n \geqslant 0} N_{n}(x) \frac{t^{n}}{n !}
$$


Using (23), the formal power series $N(x, t)$ satisfies the following partial differential equation:

$$
(1-2 x t) \frac{\partial N(x, t)}{\partial t}-2 x(1-x) \frac{\partial N(x, t)}{\partial x}=x N(x, t) .
$$

By the method of characteristics [19], it is easy to derive an explicit formula:

$$
N(x, t)=e^{x t} \sqrt{\frac{1-x}{e^{2 x t}-x e^{2 t}}} .
$$

Hence

$$
N^{2}(x, t)=\frac{1-x}{1-x e^{2 t(1-x)}} .
$$

Combining (3) and (25), we get the following result.

Theorem 9. For $n \geqslant 0$, we have

$$
\sum_{k=0}^{n}\left(\begin{array}{l}
n \\
k
\end{array}\right) N_{k}(x) N_{n-k}(x)=2^{n} A_{n}(x) .
$$

In the final part of this section, we present both central and local limit theorems for the coefficients of $N_{n}(x)$. As an application of a result [13, Theorem 2] on polynomials with only real zeros, the recurrence relation (23) enables us to show that the polynomials $\left\{N_{n}(x)\right\}_{n \geqslant 1}$ form a Sturm sequence.

Proposition 10. For $n \geqslant 2$, the polynomial $N_{n}(x)$ has $n$ distinct real zeros, separated by the zeros of $N_{n-1}(x)$.

Let $\{a(n, k)\}_{0 \leqslant k \leqslant n}$ be a sequence of positive real numbers. It has no internal zeros if there are no three indices $i<j<k$ such that $a(n, i) a(n, k) \neq 0$ and $a(n, j)=0$. Let $A_{n}=\sum_{k=0}^{n} a(n, k)$. We say that the sequence $\{a(n, k)\}$ satisfies a central limit theorem with mean $\mu_{n}$ and variance $\sigma_{n}^{2}$ provided

$$
\limsup _{n \rightarrow+\infty, x \in \mathbb{R}}\left|\sum_{k=0}^{\mu_{n}+x \sigma_{n}} \frac{a(n, k)}{A_{n}}-\frac{1}{\sqrt{2 \pi}} \int_{-\infty}^{x} e^{-\frac{t^{2}}{2}} d t\right|=0 .
$$

The sequence satisfies a local limit theorem on $B \subseteq \mathbb{R}$ if

$$
\limsup _{n \rightarrow+\infty, x \in B}\left|\frac{\sigma_{n} a\left(n, \mu_{n}+x \sigma_{n}\right)}{A_{n}}-\frac{1}{\sqrt{2 \pi}} e^{-\frac{x^{2}}{2}}\right|=0 .
$$

Recall the following Bender's theorem.

Theorem 11. [2] Let $\left\{P_{n}(x)\right\}_{n \geqslant 1}$ be a sequence of polynomials with only real zeros. The sequence of the coefficients of the polynomial $P_{n}(x)$ satisfies a central limit theorem with

$$
\mu_{n}=\frac{P_{n}^{\prime}(1)}{P_{n}(1)} \quad \text { and } \quad \sigma_{n}^{2}=\frac{P_{n}^{\prime}(1)}{P_{n}(1)}+\frac{P_{n}^{\prime \prime}(1)}{P_{n}(1)}-\left(\frac{P_{n}^{\prime}(1)}{P_{n}(1)}\right)^{2},
$$

provided that $\lim _{n \rightarrow \infty} \sigma_{n}^{2}=+\infty$. If the sequence of coefficients of the polynomial $P_{n}(x)$ has no internal zeros, then the sequence of coefficients satisfies a local limit theorem. 
Combining Proposition 10 and Theorem 11, we obtain the following result.

Theorem 12. The sequence $\{N(n, k)\}_{1 \leqslant k \leqslant n}$ satisfies a central and a local limit theorem with $\mu_{n}=(2 n+1) / 4$ and $\sigma_{n}^{2}=(2 n+1) / 24$ for $n \geqslant 4$.

Proof. By differentiating (23), we obtain the recurrence $x_{n+1}=(2 n+1) ! !+(2 n-1) x_{n}$ for $x_{n}=N_{n}^{\prime}(1)$, and this has the solution $x_{n}=(2 n+1) ! ! / 4$ for $n \geqslant 2$. By Theorem 11 , we have $\mu_{n}=(2 n+1) / 4$. Another differentiation leads to the recurrence

$$
y_{n+1}=\frac{(2 n+1) ! !}{4}(4 n-2)+(2 n-3) y_{n}
$$

for $y_{n}=N_{n}^{\prime \prime}(1)$. Set $y_{n}=(2 n-1) ! !\left(a n^{2}+b n+c\right)$ and solve for $a, b, c$ to get

$$
y_{n}=(2 n-1) ! !\left(12 n^{2}-8 n-7\right) / 48
$$

for $n \geqslant 4$. Hence $\sigma_{n}^{2}=(2 n+1) / 24$. Thus $\lim _{n \rightarrow \infty} \sigma_{n}^{2}=+\infty$ as desired.

Let $P(x)=\sum_{i=0}^{n} a_{i} x^{i}$ be a polynomial. Let $m$ be an index such that $a_{m}=\max _{0 \leqslant i \leqslant n} a_{i}$. Darroch [5] showed that if $P(x) \in \mathrm{RZ}(-\infty, 0]$, then

$$
\left\lfloor\frac{P_{n}^{\prime}(1)}{P_{n}(1)}\right\rfloor \leqslant m \leqslant\left\lceil\frac{P_{n}^{\prime}(1)}{P_{n}(1)}\right\rceil .
$$

So the following result is immediate.

Corollary 13. If $i=\lfloor(2 n+1)\rfloor / 4$ or $i=\lceil(2 n+1)\rceil / 4$ then $N(n, i)=\max _{1 \leqslant k \leqslant n} N(n, k)$.

\section{Acknowledgments}

The author thanks the referee for many detailed suggestions leading to substantial improvement of this paper. He thanks Prof. Y. Wang for his kind assistance.

\section{References}

[1] D. André, Développement de sec x et tan x, C. R. Math. Acad. Sci. Paris., 88:965967, 1879.

[2] E.A. Bender, Central and local limit theorems applied to asymptotic enumeration, J. Combin. Theory Ser. A, 15:91-111, 1973.

[3] L. Carlitz and R. Scoville, Tangent numbers and operators, Duke Math. J., 39:413429, 1972.

[4] D. Cvijović, Derivative polynomials and closed-form higher derivative formulae, Appl. Math. Comput., 215:3002-3006, 2009.

[5] J.N. Darroch, On the distribution of the number of successes in independent trials, Ann. Math. Statist., 35:1317-1321, 1964. 
[6] K. Dilks, T.K. Petersen, J.R. Stembridge, Affine descents and the Steinberg torus, Adv. in Appl. Math., 42:423-444, 2009.

[7] N. Eriksen, H. Eriksson and K. Eriksson, Diagonal checker-jumping and Eulerian numbers for color-signed permutations, Electron. J. Combin., 7 (2000), \#R3.

[8] G.R. Franssens, Functions with derivatives given by polynomials in the function itself or a related function, Anal. Math., 33:17-36, 2007.

[9] M.E. Hoffman, Derivative polynomials for tangent and secant, Amer. Math. Monthly, 102:23-30, 1995.

[10] M.E. Hoffman, Derivative polynomials, Euler polynomials, and associated integer sequences, Electron. J. Combin., 6 (1999), \#R21.

[11] M. Josuat-Vergès, Enumeration of snakes and cycle-alternating permutations, arXiv:1011.0929v1.

[12] D.H. Lehmer, Interesting Series Involving the Central Binomial Coefficient, Amer. Math. Monthly, 92:449-457, 1985.

[13] S.-M. Ma, Y. Wang, q-Eulerian polynomials and polynomials with only real zeros, Electron. J. Combin., 15 (2008), \#R17.

[14] S.-M. Ma, Derivative polynomials and enumeration of permutations by number of interior and left peaks, Discrete Math., 312(2):405-412, 2012.

[15] S.-M. Ma, An explicit formula for the number of permutations with a given number of alternating runs, J. Combin. Theory Ser. A, 119:1660-1664, 2012.

[16] E. Neuwirth, Recursively defined combinatorial functions: Extending Galton's board, Discrete Math., 239:33-51, 2001.

[17] N.J.A. Sloane, The On-Line Encyclopedia of Integer Sequences, http://oeis.org.

[18] R.P. Stanley, A Survey of Alternating Permutations, in Combinatorics and Graphs, R. A. Brualdi et. al. (eds.), Contemp. Math., Vol. 531, Amer. Math. Soc., Providence, RI, 2010, pp. 165-196.

[19] H. Wilf, The method of characteristics, and 'problem 89' of Graham, Knuth, and Patashnik, arXiv:math/0406620v2. 